\title{
Conhecimento e fonte de informações de pessoas surdas sobre saúde e doença
}

\author{
Yanik Carla Araújo de Oliveira(a) \\ Suely Deysny de Matos Celino ${ }^{(\text {b) }}$ \\ Inácia Sátiro Xavier de França(c) \\ Lorita Marlena Freitag Pagliuca(d) \\ Gabriela Maria Cavalcanti Costa(e)
}

Oliveira YCA, Celino SDM, França ISX, Pagliuca LMF, Costa GMC. Deaf people's knowledge and information sources regarding health and disease. Interface (Botucatu). 2015; 19(54):549-60.

The aim of this study was to comprehend deaf people's knowledge of health and disease and their information sources. It was a descriptive study with a qualitative approach, conducted in a public referral institution for caring for people with disabilities in the state of Paraíba. Interviews were conducted individually, in sign language, with 11 deaf people who were chosen using the speech content saturation method. The data were analyzed using content analysis. From the discourse, the following categories emerged: healthdisease process and health information sources. The deaf people interviewed in this study presented limited knowledge of the health-disease process. The subjects placed value on doctors as indispensable to the health-disease process. Some deaf people displayed lack of knowledge about health because of poor and inefficient sources that provide primary information without depth of content, and therefore demanded greater opportunities.

Keywords: Deafness. Health-disease process. Access to information. Health education.
Objetivou-se compreender o conhecimento de pessoas surdas em saúde e doença e suas fontes de informações em um estudo descritivo, com abordagem qualitativa, realizado em uma instituição pública de referência, no estado da Paraíba, em atendimento a pessoas com deficiência. Foram realizadas entrevistas individualmente, em língua de sinais, com 11 pessoas surdas escolhidas pelo método de saturação do conteúdo das falas. Os dados foram analisados segundo a análise de conteúdo. Das falas, emergiram as categorias: processo saúde-doença e fontes de informações de saúde. Os surdos entrevistados nesse estudo apresentaram um conhecimento limitado sobre o processo saúde-doença. Os sujeitos valorizam o médico como imprescindível para o processo saúde-doença. Alguns surdos expuseram não conhecer sobre saúde, devido a fontes precárias e pouco eficientes, disponibilizando informações primárias, sem profundidade de conteúdos, e, por isso, reivindicam mais oportunidades.

Palavras-chave: Surdez. Processo saúdedoença. Acesso à Informação. Educação em Saúde. (a) Fisioterapeuta. AE04, módulo J, Residencial Sports Club, torre II, apto. 2003, Guará II. Brasília, DF, Brasil. 71070704.yanikaraujo@ yahoo.com.br (b) Faculdade de Ciências Médicas de Campina Grande. Campina Grande, PB, Brasil. deysny@hotmail.com (c,e) Universidade Estadual da Paraíba. Campina Grande, PB, Brasil. inacia.satiro@ gmail.com; gabymcc@ bol.com.br

(d) Universidade Federal do Ceará. Fortaleza, CE, Brasil.pagliuca@ufc.br 


\section{Introdução}

As concepções sobre a saúde e a doença têm sido construídas, ao longo do tempo, a partir do desenvolvimento teórico-conceitual da ciência e, ainda mais, por condicionantes ideológicos que influenciam conceitos mais legítimos em detrimento de outros. Conceitos que perpassaram desde as crenças mágico-religiosas, o empirismo racional, até a ciência moderna, e retrataram as transformações de inferência causal sobre o processo de adoecimento ao longo da história¹.

Apesar de a Organização Mundial de Saúde, desde 1948, já ter introduzido o conceito de saúde como um completo bem-estar físico, mental e social, a ideia de saúde como ausência de doença, fundamentada no paradigma biomédico, por muito tempo, foi amplamente difundida pela medicina, e, ainda hoje, orienta não só o senso comum, como, também, diversas pesquisas e produção tecnológica em saúde, sobretudo as relacionadas aos avanços na área de diagnóstico².

A Carta de Otawa, elaborada em 1986 na I Conferência Internacional sobre Promoção da Saúde, associa o conceito de saúde ao de qualidade de vida, influenciado por fatores políticos, econômicos, sociais, culturais, ambientais, comportamentais e biológicos ${ }^{3}$. Também a Lei $n^{\circ}$ 8.080/1990, que regulamenta o Sistema Único de Saúde (SUS) em nosso país, incorporou esse conceito, entendendo, ainda, a saúde como direito fundamental do ser humano ${ }^{4}$.

Nesse sentido, prestar assistência à saúde vai muito além das atividades clínicas, suscitando a necessidade de ações no âmbito de todas as condições que abrangem o processo saúde-doença, compreendido como "um processo social caracterizado pelas relações dos homens com a natureza e com outros homens, num determinado espaço geográfico e num determinado tempo histórico" ${ }^{5}$ (p. 11).

A partir desses conceitos, entende-se que, dentre outros fatores, o nível de conhecimento de uma população influencia diretamente no processo saúde-doença de diversas formas: na percepção dos problemas de saúde, na capacidade de entendimento das informações sobre saúde, na adoção de estilos de vida saudáveis; no consumo e utilização dos serviços de saúde, e na adesão aos procedimentos terapêuticos ${ }^{6}$.

Dessa forma, destaca-se a importância das práticas educativas realizadas pelos profissionais de saúde no sentido de sensibilizar a população para a transformação da situação de saúde da sociedade brasileira, a partir de ações que estimulem não só uma nova consciência sanitária, mas a democratização de políticas públicas, contribuindo, então, para aumentar a visibilidade dos sujeitos sobre sua inserção histórica, social e política, e levando suas enunciações e reivindicações? ${ }^{7}$.

No caso das pessoas surdas, as informações em saúde tornam-se muitas vezes limitadas pela dificuldade de comunicação dos profissionais com esses usuários. Além disso, uma vez que possuem privações linguísticas, os surdos também apresentam dificuldade de aprendizagem da língua portuguesa escrita, o que pode acarretar limitações cognitivas, sociais, educacionais e culturais ${ }^{8}$.

Nesse sentido, a Lei de Acessibilidade $n^{\circ} 10.098$, de 2000, atribuiu, como responsabilidade do poder público, o dever de promover a eliminação de barreiras na comunicação e estabelecer mecanismos e alternativas técnicas que tornem acessíveis os sistemas de comunicação e sinalização às pessoas com deficiência sensoriais e com dificuldade de comunicação?.

Dessa forma, este trabalho teve por objetivo compreender o conhecimento de pessoas em saúde e doença e suas fontes de informações.

\section{Caminho metodológico}

Trata-se de um estudo descritivo-analítico, com abordagem qualitativa, realizado em uma instituição pública de referência, no estado da Paraíba, em atendimento a pessoas com deficiência, localizada em João Pessoa/PB.

A instituição foi escolhida por desenvolver trabalho na inserção, articulação e fortalecimento da comunidade surda na Paraíba, tendo sido esclarecido, aos colaboradores, que a eleição do cenário serviu apenas para localizar os sujeitos, e que os questionamentos sobre os serviços de saúde pública não se relacionavam aos serviços prestados pela instituição. 
Participaram do estudo 11 pessoas com surdez que aguardavam atendimento nos serviços da instituição, respeitando os critérios de inclusão determinados, ou seja: ter acima de 18 anos de idade; se comunicar em língua de sinais; apresentar condição cognitiva que permita a interação com a pesquisadora bilíngue, e assinar o Termo de Consentimento Livre e Esclarecido (TCLE), atestando o esclarecimento sobre os objetivos da pesquisa, aceitando voluntariamente sua participação.

Foram realizadas entrevistas individuais, considerando a disponibilidade e conveniência do entrevistado. A comunicação das entrevistas ocorreu por meio da língua de sinais, por ser a língua dos participantes, e, devido à fluência da pesquisadora, em Libras, foi estabelecido contato direto com os surdos, mantendo a privacidade das informações. Diante do uso de uma comunicação espaço-visual, as entrevistas foram filmadas em arquivo MP4.

Para a condução das entrevistas, foi utilizado um roteiro, com dados socioeconômicos e questões norteadoras: O que você entende por saúde? O que você entende por doença? Como você faz para obter informações sobre saúde?

As falas dos entrevistados foram traduzidas para o português e transcritas pela própria pesquisadora. Em seguida, os vídeos e as transcrições foram entregues a um juiz surdo bilíngue para comprovação das traduções. Considerando a dificuldade em identificar um profissional qualificado em tradução Libras-Português com domínio pleno nos dois idiomas, a averiguação das traduções limitou-se, portanto, a um único profissional surdo. Esse processo era realizado ao final de cada dia de entrevistas, durante o período de coleta dos dados. O número de sujeitos baseou-se no critério de saturação do conteúdo das falas.

Os dados foram analisados de acordo com a análise de conteúdo temática, proposta por Bardin ${ }^{10}$, que estabelece um conjunto de técnicas de análise da comunicação verbal com a descrição do conteúdo das mensagens dos entrevistados. Seu método é composto de três fases (pré-análise, exploração do material e tratamento dos resultados, inferência e interpretação), e a categorização dos dados aconteceu agrupando os temas que contemplam o mesmo significado.

A pesquisa foi autorizada pelo Comitê de Ética em Pesquisa da Universidade Estadual da Paraíba, e registra-se o cumprimento dos princípios bioéticos postulados na Resolução 466/2012, do Conselho Nacional de Saúde. Dessa forma, para preservar o anonimato dos sujeitos, foi registrado um código no qual cada planta do bioma nativo brasileiro corresponde a um participante.

\section{Resultados e discussões}

Houve um equilíbrio com relação ao sexo dos entrevistados, sendo sete do sexo masculino e quatro do feminino, com idade média de 30,6 anos. No que se refere ao nível educacional, um possuía Ensino Fundamental incompleto, um possuía Ensino Superior e nove possuíam Ensino Médio completo. Houve um predomínio de sujeitos com quatro ou mais familiares no domicílio (oito), com renda familiar prevalente entre um e dois salários-mínimos (seis), e apenas seis indivíduos afirmaram ter algum familiar, sem considerar o cônjuge, que sabe língua de sinais.

Das falas dos entrevistados, foram estabelecidas as seguintes categorias temáticas: processo saúdedoença e informações sobre saúde.

\section{Processo saúde-doença}

A expressão saúde incorporou diversos sentidos que variaram com a época e com os grupos sociais, e, por muito tempo, foi colocada em oposição ao conceito de doença, até a compreensão de que esses dois termos, apesar de se tratarem de coisas diferentes, são, ao mesmo tempo, indissociáveis; e, sendo assim, a saúde se manifesta como uma constante busca de equilíbrio para superar o processo de adoecimento ${ }^{11}$.

Todas as atitudes na área da saúde, desde o diagnóstico clínico até questões legais referentes aos direitos no âmbito da saúde, pautam-se em um entendimento simples e, ao mesmo tempo, complexo do que é ou do que significa o 'ser saudável'12. 
Apesar disso, para a pessoa com surdez, o acesso às informações relacionadas à saúde pode não ser o mesmo da população em geral, quando os surdos não têm acesso a informações de conversas ouvidas por acaso, ou anúncios de rádio ou televisão. Mesmo que sejam alfabetizados em português, a informação escrita também é limitada ${ }^{13}$. Esse fato confirma os achados deste estudo, no qual a maioria dos sujeitos, mesmo apresentando grau de escolaridade equivalente ao Ensino Médio completo, relatou não ter conhecimentos sobre o que é saúde e doença.

"Ninguém nunca me ensinou nada de saúde". (Caviúna)

"Não sei como aparece. Se me perguntar, não sei nada. Só sinto dor. Não sei como acontece". (Timbó)

"A gente não entende o que acontece". (Capixingui)

O conhecimento sobre saúde, mesmo que elementar, é imprescindível para que o sujeito possa contribuir de forma positiva no processo saúde-doença. É a partir do empoderamento de informações que as pessoas podem exercer, efetivamente, o seu direito à cidadania, e, consequentemente, à saúde ${ }^{14}$.

Nesse sentido, o Ministério da Saúde, por meio da Portaria 2.761/2013, instituiu a Política Nacional de Educação Popular em Saúde no âmbito do SUS, com o compromisso da construção de um projeto de educação democrático e popular, por uma sociedade justa, solidária, democrática, igualitária, tendo como um dos seus eixos estratégicos a formação, a comunicação e a produção de conhecimento ${ }^{15}$.

Apesar da falta de conhecimento de alguns, podem-se observar ainda sujeitos que demonstraram possuir algum tipo de entendimento a respeito do processo de adoecimento, que se baseou tanto na concepção mágico-religiosa quanto no modelo biomédico.

“Deus nos dá a saúde, a gente precisa ter fé para ter saúde, e que cada vez mais vai melhorar. Primeiro Deus dá a saúde, aí a gente luta e tem fé de continuar assim. Eu não sei como acontece, Deus é quem sabe e dá a cura". (Gurucaia)

O conceito de saúde-doença relacionado ao aspecto religioso ainda exerce forte influência na população, que atribui à doença, muitas vezes, um castigo divino, algo que se introduz no organismo humano por causa do pecado ${ }^{16}$, e, ao contrário, a saúde como dádiva divina, como expressado pela fala acima.

A explicação para a relação da religiosidade e a comunidade surda está na história dessa comunidade no Brasil, onde os grupos religiosos se apresentaram como solidários à condição dos surdos, disseminando a língua de sinais entre os ouvintes que se interessassem, e ampliando seus conhecimentos sobre a cultura surda ${ }^{17}$. Para evitar o isolamento das pessoas surdas, instituições com fins religiosos, educativos e sociais dispunham de alguns serviços para a comunidade surda, como o de interpretação de língua de sinais ${ }^{18}$.

Algumas pessoas ainda entendem seu processo de adoecimento como uma tendência natural ou instintiva de um sujeito com um problema de saúde, ou de qualquer um que se ponha no seu lugar, e associe esse fato com um mal indesejável que the aflige e lhe impede de realizar as principais atividades de sua vida. As informações que os sujeitos têm são sobre o fato físico que afeta a funcionalidade de algum de seus órgãos e acaba lhes gerando um desconforto pessoal ou, mesmo, social ${ }^{12}$, conforme falas a seguir.

“Existem dois tipos: um é aquele evitável, precisa cuidar da saúde, comer certo; ter cuidado para não engordar e coisas assim. Outra coisa é quando aparece de repente, vem de dentro do corpo e se espalha. Então são dois tipos: um evitável e outro que surge de repente". (Monjoleiro) 
"Tem uma doença simples que é fácil, mas também tem a doença profunda, perigosa que eu não conheço bem, não. A doença simples tem a febre, manchas no corpo, uma dor de barriga. Mas eu não sei como acontece". (Gurucaia)

"Coisas de saúde... resfriado, dor de garganta, dor de barriga, alergia e coisas do tipo". (Mutambo)

Essa visão reducionista também foi encontrada em estudo com adolescentes de Ensino Fundamental, que atribuíam à doença apenas a disfunções do corpo físico ${ }^{19}$. Essa forma de entender a saúde reflete o conhecimento generalizado da população baseado no modelo médico-assistencial privatista, voltado para os indivíduos que procuram os serviços de saúde quando estão doentes ${ }^{20}$, pelo entendimento da saúde como ausência de doença.

Ainda nesse sentido, alguns dos entrevistados, segundo as falas a seguir, evidenciaram o atendimento médico não só como importante, mas satisfatório e suficiente para resolver seus problemas de saúde.

“Quando tem alguma coisa errada no corpo, daí a gente vai ao médico mostra para ele descobrir o que é e nos dar o remédio para nos curar, nos proteger e resolver o problema. (Jacarandá)

Quando você vai ao médico, acaba com a doença e fica com saúde. Só indo ao médico, para curar as doenças, evitar algumas coisas que piorem a saúde, aprender coisas que são perigosas para saúde. Só indo ao médico para saber essas coisas". (Maricá)

“Eu falo sobre a importância de ir ao médico para investigar sobre como está a saúde. Ele faz uma avaliação da nossa saúde... O surdo precisa ir ao médico saber como andam as coisas no próprio corpo, se está tudo bem por dentro. Ele passa remédio para gente melhorar, e com um intérprete para pode explicar tudo direitinho, combina bem. A gente segue com saúde". (Capixingui)

“Uma vez eu fui ao médico sozinho e disse que estava sentindo a minha pressão alta, o médico mediu e confirmou. Ele me disse que eu precisava tomar um remédio embaixo da língua, depois de meia hora eu fui ao banheiro várias vezes e a pressão baixou. Ele mediu de novo e fui para casa". (Urucum)

Destaca-se que o participante identificado como Urucum, relatou uma experiência de atendimento de emergência, declarando ter 'resolvido seu problema de saúde', contudo, observa-se que o mesmo não recebeu nenhuma orientação acerca da sua condição, nem mesmo demonstrou conhecimentos sobre que medicação fez uso.

O usuário deveria ter sido orientado sobre a necessidade de aferir a pressão arterial por mais duas vezes, para que o diagnóstico de hipertensão arterial pudesse ser confirmado e seguido o acompanhamento, além de informações sobre seus fatores de risco modificáveis, como: sobrecarga de ingestão de sal, excesso de adiposidade, abuso de álcool, entre outros ${ }^{21}$.

Esse fato traz preocupações sobre as consequências da desinformação sobre saúde ou doença das pessoas surdas e de quanto isso influencia negativamente seus cuidados de saúde. Observa-se, dessa forma, que não foram encontrados sujeitos que discorressem sobre o conceito de saúde de forma ampla, determinada por diversos fatores que a condicionam, como: moradia, educação, renda e acesso aos serviços essenciais, o que está assegurado na legislação brasileira ${ }^{4}$.

Esse achado diverge de outros estudos realizados com usuários de serviços de saúde da atenção primária, nos quais as respostas dos entrevistados, mesmo não possuindo a ideia clara de determinantes sociais, apresentaram visão ampliada do conceito de saúde, congruente com os pressupostos do modelo assistencial proposto pela Reforma Sanitáriaa ${ }^{22,23}$. 


\section{Informações em saúde}

A diversidade de tecnologias de comunicação, especialmente a internet, grandemente difundida para a maioria da população, tem facilitado o acesso à informação em saúde, exercendo uma influência enorme sobre a situação de saúde e, em especial, sobre as iniquidades em saúde ${ }^{6}$, visto que muitas informações, que eram limitadas a um determinado grupo, estão abertas a todas as pessoas.

Nesse sentido, a Convenção sobre os Direitos das Pessoas com Deficiência entende que essas pessoas também devem desfrutar dos mesmos meios de acesso às informações; e, portanto, a fim de assegurar-lhes esse direito, determinou que devem ser promovidas formas apropriadas de atendimento e apoio a pessoas com deficiência, para aproximá-las de todos os sistemas e tecnologias da informação e comunicação dos quais o restante da população dispõe ${ }^{24}$.

Baseado nesse pensamento, recomenda-se traduzir as informações em saúde para a língua de sinais, e adaptar a apresentação dessa informação para que seja culturalmente apropriada aos indivíduos. Informações de saúde acessíveis e culturalmente apropriadas podem ajudar os usuários da língua de sinais, os surdos, a tomarem decisões sobre comportamentos de saúde associados com riscos de doenças crônicas ${ }^{25}$.

Os relatos dos sujeitos apontam a mãe como a figura familiar mais marcante para o aprendizado sobre saúde. A maioria dos entrevistados que referiram ter algum parente que sabe se comunicar em Libras destacou a mãe como pessoa com quem consegue a melhor comunicação. E isto é confirmado pelas falas a seguir:

\footnotetext{
"Sempre pergunto a minha mãe e ela me explica tudo eu aprendo. Sou muito curiosa. Quando acontece alguma coisa com alguém eu sempre quero saber o que e como aconteceu, pergunto a minha mãe e ela descobre e me fala". (Maricá)

"Minha mãe sempre me explicou coisas de saúde... minha mãe interpreta tudo para mim". (Mutambo)
}

Geralmente, são os familiares das pessoas surdas que exercem a função de mediador entre eles e os chamados ouvintes ${ }^{18}$. Quando as mães se conscientizam das necessidades linguísticas de seus filhos surdos, tendem a usar estratégias para uma comunicação de forma efetiva ${ }^{11}$.

Em se tratando da qualidade das interações entre a pessoa surda e os membros da família, Alves ${ }^{26}$ coloca que os familiares precisam estimular as potencialidades desses sujeitos, para que, de fato, eles possam construir oportunidades reais de comunicação com o mundo exterior.

Apesar do importante papel da família e, em especial da figura da mãe, como provedora de informações de saúde, sabe-se da responsabilidade dos profissionais de saúde em orientar e informar a todos da população sobre os cuidados em saúde, para prevenção e tratamento adequado.

No contexto da educação popular em saúde, sobretudo, o usuário deve ser reconhecido pelo profissional como sujeito ativo, dotado de conhecimento sobre o seu processo saúde-doença-cuidado e capaz de desenvolver uma análise crítica sobre suas necessidades ${ }^{27}$. Ao despertar autonomias individuais e coletivas, a educação em saúde contribui para a ampliação do significado dos direitos de cidadania, favorecendo, ainda, a comunicação em variadas linguagens, transformando as informações em instrumentos para a produção de mudanças ${ }^{28}$.

As pessoas surdas sentem a necessidade de inclusão em diversas atividades desenvolvidas para a população no geral, basicamente em ações educativas que buscam fornecer informações em saúde essenciais, como apresentado na fala:

"É muito importante que os surdos participem de palestras, com intérprete, para aprender e entender com clareza". (Mutambo)

As ações educativas nos serviços de saúde almejam a garantia de uma assistência integral e a produção de saberes coletivos, propiciando, ao usuário, autonomia e capacidade de cuidar de si 
próprio e do outro. Nesse sentido, a educação permanente dos profissionais se concretiza como uma ferramenta indispensável para que as práticas educativas estejam cada vez mais adequadas às necessidades da população 29 .

Dessa forma, a legislação brasileira, reconhecendo as especificidades comunicativas da comunidade surda, por meio do Decreto 5.696 de 2005, orienta que os atendimentos em serviços públicos de saúde devam ser prestados por profissionais capacitados para o uso de Libras ou para sua tradução e interpretação ${ }^{30}$.

Também o Ministério da Saúde, por meio da Política Nacional de Saúde da Pessoa com Deficiência, tem buscado garantir acessibilidade aos serviços de saúde a esse grupo populacional; e, dessa forma, faz-se necessário que esses estabelecimentos sejam adaptados em seu aspecto físico e ambiental relacionados à comunicação, bem como a capacitação dos recursos humanos ${ }^{31}$.

Kritzinger ${ }^{32}$ afirma que surdos e deficientes auditivos têm menor probabilidade de receber informações preventivas de profissionais de saúde ou da mídia, e maior probabilidade de receber informações da comunidade surda, assim como o relato do colaborador Monjoleiro:

"A televisão sempre mostra muita informação sobre saúde, mas não tem intérprete. Na televisão eu sei que tem um programa sobre saúde, que mostra e ensina as coisas, mas falta o intérprete no quadrinho para traduzir para língua de sinais. Por isso continuamos ignorantes... Como eu sozinho vou entender que comida evitar, que cuidados tomar com o meu corpo?". (Monjoleiro)

Scheier ${ }^{33}$ aponta que pessoas surdas têm acesso limitado a materiais educativos e, por isso, confiam em seus colegas surdos para informações de saúde, o que reforça a desinformação.

A Lei no 10.098/2000, que estabelece normas e critérios para a promoção da acessibilidade das pessoas portadoras de deficiência, determina, como barreiras nas comunicações, "qualquer entrave ou obstáculo que dificulte ou impossibilite a expressão ou o recebimento de mensagens por intermédio dos meios ou sistemas de comunicação, sejam ou não de massa"9 (p. 1). Assim, é necessária a adaptação aos meios de comunicação e de massa, como a interpretação em Libras de programas informativos sobre saúde, apontados por Monjoleiro.

Esse processo de adaptação já foi regulamentado pelo Decreto nº 5.296/2004 que, em seu Art. 52, responsabiliza o Poder Público pelo incentivo da "oferta de aparelhos de televisão equipados com recursos tecnológicos que permitam sua utilização de modo a garantir o direito de acesso à informação às pessoas portadoras de deficiência auditiva ou visual" ${ }^{34}$ (p. 3). Essa legislação garante, à pessoa surda, que a mensagem seja veiculada na televisão, entre outros sistemas, por meio da janela com intérprete de Libras.

Ainda é importante ressaltar o papel da escola na informação em saúde. Costa et al. ${ }^{35}$ consideram esse espaço como fundamental para influenciar a adoção de estilos de vida saudáveis e evitar comportamentos nocivos. Enfatizam, ainda, que a vigilância em saúde de crianças e jovens no ambiente escolar complementa-se com o desenvolvimento de programas direcionados aos problemas de saúde prioritários.

Reconhecendo essa realidade, o Ministério da Saúde, articulado ao Ministério da Educação, criou, em 2007, o Programa Saúde na Escola, que tem como objetivo, entre outros, "promover a saúde e a cultura de paz, reforçando a prevenção de agravos à saúde e articular as ações da rede pública de saúde com as ações da rede pública de Educação Básica" ${ }^{36}$ (p. 12).

Apesar de todo o esforço no sentido de tornar acessível as informações em saúde, observa-se a limitação dos sujeitos do estudo no que se refere à compreensão de alguns aspectos relacionados à saúde, adquiridos nos livros ou na faculdade.

"Eu vejo nos livros, sobre como evitar, coisas assim, mas não sei nada mais profundo não. A leitura é bem difícil". (Barbatimão) 
"O que eu conheço sobre saúde, é sobre a AIDS na escola eles explicaram sobre como pega essa doença e eu aprendi. Só... Outros lugares para aprender sobre saúde não tem. Só sei sobre como evitar Aids, drogas, essas coisas. Eu aprendi que é proibido. Eu aprendi aqui na FUNAD, e fora, na universidade". (Mutambo)

Em estudo realizado com alunos surdos de Ensino Superior, observou-se dificuldade dos entrevistados com a língua portuguesa, no referente à leitura e compreensão dos textos, sendo essa limitação apresentada como barreira para o domínio dos conteúdos e o desenvolvimento do raciocínio ${ }^{37}$.

Essa população precisa de materiais cultural e linguisticamente alinhados para atendê-la de forma satisfatória, ou seja, adaptados para os formatos mais adequados às pessoas surdas que só se comunicam por meio da língua de sinais. Isso repercute na compreensão de doenças, instruções de uso de medicamentos, e acesso à informação em saúde-educação em revistas, jornais e programas de televisão legendados ${ }^{32}$.

Apesar de os entrevistados apresentarem média elevada de grau de instrução, conforme caracterização dos sujeitos, detêm baixo nível de informações, com fontes limitadas. Grande parte deles referiu não ter acesso a fontes de informações disponíveis para aprenderem sobre saúde, expuseram sua fragilidade quanto ao aprendizado sobre saúde, apontando que essa é uma realidade de outras pessoas surdas, agravada pela falta de comunicação no âmbito familiar.

"Não sei nada... Meus amigos não sabem nada. Na minha família não tem comunicação... o que eu sei é zero! Não há como a gente aprender". (Monjoleiro)

"Não tem como... Eu não aprendi nada!". (Bugreiro)

"Não sei nada". (Timbó)

Kritzinger ${ }^{32}$ considera o fato de que pessoas surdas, muitas vezes, têm as informações por meio da própria comunidade surda, e que, embora isso possa ser um meio eficaz de transferência de informação, é uma fonte informal que nem sempre é precisa e pode levar à desinformação e às lacunas no conhecimento. Por isso, alerta para a importância de se treinar e qualificar pessoas surdas a se tornarem educadores e comunicadores sociais capazes de fornecer informações sobre cuidados de saúde.

\section{Considerações finais}

Os surdos entrevistados neste estudo apresentaram um conhecimento limitado sobre o processo saúde-doença, atribuindo-o, sobretudo, ao aspecto religioso, ou como conceitos controversos. Este fato traz repercussões diversas para a qualidade de vida dos indivíduos, como: a falta de protagonismo quanto ao autocuidado de saúde, falta de informações pela dificuldade em acessar fontes de informações, e a dependência de ouvintes, familiares, amigos e intérpretes de Libras, devido à barreira da comunicação. Mesmo assim, observou-se que os sujeitos seguem o modelo biomédico centrando a saúde na ausência de doenças, por meio da valorização do médico como sendo imprescindível para o processo saúde-doença, na cura das doenças.

Dessa forma, os surdos sentem dificuldade em promover cuidados preventivos de saúde, e buscar serviços nesse sentido ou seguir adequadamente um tratamento de saúde, devido à barreira da comunicação, causada pelo despreparo dos profissionais em reconhecer a cultura surda e suas necessidades comunicativas em língua de sinais.

Quando se trata de informações de saúde, encontrou-se um quadro ainda mais inquietante, no qual as fontes de informações para os indivíduos surdos são precárias e pouco eficientes, disponibilizando apenas informações primárias, sem profundidade de conteúdos. Apontaram que a 
comunicação em meios de massa, como a televisão, ainda é inalcançável para os surdos, limitando seu acesso a informações que são disponíveis para ouvintes de todas as idades.

Mesmo tendo um nível educacional acima do Ensino Médio, os surdos necessitam de adequações em materiais educativos e informativos, considerando as características da cultura surda e da língua de sinais, de modo a promover uma igualdade de oportunidade de aprendizado. Destarte, sugere-se que a educação em saúde, tão difundida por ações de prevenção e promoção da saúde, possa ser adaptada a pessoas que se comunicam em língua de sinais, para que elas possam adquirir o conhecimento necessário a fim de cuidar plenamente de sua própria saúde e tomar suas decisões, nesse sentido, com autonomia.

A educação em saúde deve ser pensada e executada, tanto pelas escolas, enquanto educadoras, quanto pelos profissionais de saúde, enquanto cuidadores da saúde. Por isso, a formação profissional e o serviço público devem incorporar, às suas práticas, a sensibilização dos estudantes e profissionais quanto à cultura surda e suas repercussões na vida dos surdos, bem como as estratégias de minimizar os obstáculos provenientes dessa surdez.

\section{Colaboradores}

Yanik Carla Araújo de Oliveira e Gabriela Maria Cavalcanti Costa participaram da concepção do projeto, análise e interpretação dos dados; redação do artigo e revisão crítica relevante do conteúdo intelectual; e aprovação final da versão a ser publicada. Suely Deysny de Matos Celino participou da análise e interpretação dos dados; redação do artigo e revisão crítica relevante do conteúdo intelectual; e aprovação final da versão a ser publicada. Inácia Sátiro Xavier de França e Lorita Marlena Freitag Pagliuca participaram da análise e interpretação dos dados; revisão crítica relevante do conteúdo intelectual; e aprovação final da versão a ser publicada.

\section{Referências}

1. Oliveira MAC, Egry EY. A historicidade das teorias interpretativas do processo saúdedoença. Rev Esc Enferm USP. 2000; 34(1):9-15.

2. Batistella C. Abordagens contemporâneas do conceito de saúde. In: Fonseca AF, Corbo $A D$, organizadores. O território e o processo saúde-doença. Rio de Janeiro: EPSJV, Fiocruz; 2007. p. 51-86.

3. Carta de Otawa. Primeira Conferência Internacional sobre Promoção da Saúde [Internet]. 1986 [acesso 2010 Ago 18]. Disponível em: http://www.opas.org.br/ promocao/uploadArq/Ottawa.pdf

4. Lei no 8.080 , de 19 de setembro de 1990. Dispõe sobre as condições para a promoção, proteção e recuperação da saúde, a organização e o funcionamento dos serviços correspondentes e dá outras providências. Diário Oficial da União. 19 Set 1990. 
5. Tancredi FB, Barrios SRL, Ferreira JHG. Planejamento em saúde. São Paulo: Faculdade de Saúde Pública, Universidade de São Paulo; 1998.

6. Ministério da Saúde. Comissão Nacional sobre determinantes sociais da saúde: as causas sociais das iniquidades em saúde no Brasil. Rio de Janeiro: Fiocruz; 2008.

7. Fundação Nacional de Saúde. Diretrizes de educação em saúde visando à promoção da saúde. Brasília (DF): Funasa; 2007.

8. Fernandes EL. Surdez versus aprendizado da língua portuguesa escrita. Rev CES/JF. 2008; 22(1):77-88.

9. Lei no 10.098, de 19 de dezembro de 2000. Estabelece normas gerais e critérios básicos para a promoção da acessibilidade das pessoas com deficiência ou com mobilidade reduzida, e dá outras providências. Diário Oficial da União. 20 Dez 2000.

10. Bardin L. Análise de conteúdo. São Paulo: Edições 70; 2011.

11. Ayres JRCM. Uma concepção hermenêutica da saúde. Physis. 2007; 17(1):43-62.

12. Serafim RA. Teoria da comunicação social do conceito de saúde. Bol Inst Saude. 2010; 12(1):11-5.

13. Barnett S. Communication with deaf and hard-of-hearing people: a guide for medical education. Acad Med. 2002; 77(7):694-700.

14. Leite RAF. Direito à informação: análise do conhecimento do paciente acerca de seus direitos [dissertação]. Ribeirão Preto (SP): Universidade de São Paulo; 2010.

15. Portaria $n^{\circ} 2.761 / \mathrm{GM} / \mathrm{MS}$, de 19 de novembro de 2013. Institui a Política Nacional de Educação Popular em Saúde no âmbito do Sistema Único de Saúde (PNEPS-SUS). Diário Oficial da União. 20 Nov 2013.

16. Scliar M. História do conceito de saúde. Physis. 2007; 17(1):29-41.

17. Lacerda CBF. Tradutores e intérpretes de Língua Brasileira de Sinais: formação e atuação nos espaços educacionais inclusivos. Cad Educ. 2010; 36(1):133-53.

18. Masutti ML, Santos SA. Intérpretes de Língua de Sinais: uma política em construção. In: Quadros RM, organizador. Estudos surdos III. Petrópolis: Arara Azul; 2008. p. 148-67.

19. Nery AA, Silva DR, Bueno ESG, Santos FPA, Nascimento MS, Carvalho PAL, et al. Concepção de saúde: visão de adolescentes do ensino fundamental de um município da Bahia. Rev Saude.Com. 2009; 5(1):17-30.

20. Paim JS. Modelos de atenção e vigilância em saúde. In: Rouquayrol MZ, Almeida Filho N, organizadores. Epidemiologia e saúde. 6a ed. Rio de Janeiro: Guanabara Koogan; 2003. p. 567-77.

21. Ministério da Saúde. Secretaria de Atenção à Saúde. Estratégias para o cuidado da pessoa com doença crônica: hipertensão arterial sistêmica. Brasília (DF): MS; 2013.

22. Fertonani HP, Pires D. Concepção de saúde de usuários da Estratégia Saúde da Família e novo modelo assistencial. Enferm Foco. 2010; 1(2):51-4.

23. Wendhausen ALP, Rebello BC. As concepções de saúde-doença de portadores de hipertensão arterial. Cienc Cuid Saude. 2004; 3(3):243-51.

24. Organização das Nações Unidas. Convenção sobre os Direitos das Pessoas com Deficiência. Nova York: ONU; 2006.

25. Barnett S, McKee M, Smith SR, Pearson TA. Deaf sign language users, health inequities, and public health: opportunity for social justice. Prev Chronic Dis [Internet]. 2011 [acesso 2013 Mar 11]; 8(2):A45. Disponível em: http://www.ncbi.nlm.nih.gov/

pubmed/21324259?report=docsum 
26. Alves PF. Discurso da mãe ouvinte durante a interação com o filho surdo [dissertação]. Rio de Janeiro (RJ): Universidade Veiga de Almeida; 2010.

27. Alves VS. Um modelo de educação em saúde para o Programa Saúde da Família: pela integralidade da atenção e reorientação do modelo assistencial. Interface (Botucatu). 2005; 9(16):39-52.

28. Pedroza JIL. Educação popular no Ministério da Saúde: identificando espaços e referências. In: Ministério da Saúde. Secretaria de Gestão Estratégica e Participativa. Caderno de educação popular e saúde. Brasília (DF): MS; 2007. p. 13-17.

29. Oliveira RL, Santos MEA. Educação em saúde na estratégia saúde da família: conhecimentos e práticas do enfermeiro. Rev Enferm Integr. 2011; 4(2):833-44.

30. Decreto $n^{\circ} 5.626$, de 22 de dezembro de 2005. Regulamenta a Lei no 10.436, de 24 de abril de 2002, que dispõe sobre a Língua Brasileira de Sinais. Diário Oficial da União. 23 Dez 2005.

31. Ministério da Saúde. Secretaria de Atenção à Saúde. Departamento de Ações Programáticas Estratégicas. Atenção à saúde da pessoa com deficiência no Sistema Único de Saúde. Brasília (DF): MS; 2009.

32. Kritzinger J. Exploring the barriers and facilitators to health care services and health care information for deaf people in Worcester [dissertação]. África do Sul: Universidade de Stellenbosch; 2011.

33. Scheier DB. Barriers to health care for people with hearing loss: a review of the literature. J N Y State Nurses Assoc. 2009; 40(1):4-10.

34. Decreto n० 5296, de 02 de dezembro de 2004. Regulamenta as Leis $n^{\circ} 10.048$, de 8 de novembro de 2000, que dá prioridade de atendimento às pessoas que especifica, e 10.098, de 19 de dezembro de 2000, que estabelece normas gerais e critérios básicos para a promoção da acessibilidade das pessoas portadoras de deficiência ou com mobilidade reduzida, e dá outras providências. Diário Oficial da União. 03 Dez 2004.

35. Costa GMC, Cavalcanti VM, Barbosa ML, Celino SDM, França ISX, Sousa FS. Promoção de saúde nas escolas na perspectiva de professores do ensino fundamental. Rev Eletr Enferm [Internet]. 2013 [acesso 2013 Dez 12]; 15(2):506-15. Disponível em: http://dx.doi.org/10.5216/ree.v15i2.15769

36. Ministério da Saúde. Secretaria de Gestão Estratégica e Participativa. Saúde na Escola. Brasília (DF): MS; 2009.

37. Daroque SC, Padilha AML. Alunos surdos no ensino superior: uma discussão necessária. Comunicaçoes. 2012; 19(2):23-32. 
Oliveira YCA, Celino SDM, França ISX, Pagliuca LMF, Costa GMC. Conocimiento y fuente de informaciones de personas sordas sobre salud y enfermedad. Interface (Botucatu). 2015; 19(54):549-60.

El objetivo fue comprender el conocimiento de personas sordas sobre salud y enfermedad y sus fuentes de informaciones en uno estudio descriptivo, con abordaje cualitativo, realizado en una institución pública de referencia, en el estado de Paraíba, en atención a personas con discapacidad. Se realizaron entrevistas individuales, en lengua de signos, con 11 personas sordas elegidas por el método de saturación del contenido de las conversaciones. Los datos se analizaron según el análisis de contenido. De las conversaciones, surgieron las categorías: proceso salud-enfermedad y fuentes de informaciones de salud. Los sordos entrevistados en este estudio presentaron un conocimiento limitado sobre el proceso salud-enfermedad. Los sujetos valorizan al médico como imprescindible para el proceso salud-enfermedad. Algunos sordos expusieron que no tenían conocimiento sobre salud, debido a fuentes precarias y poco eficientes, poniendo a disposición informaciones primarias, sin profundidad de contenidos y, por eso, reivindican mayores oportunidades.

Palabras clave: Sordez. Proceso salud-enfermedad. Acceso a la información. Educación en salud.

Recebido em 16/04/14. Aprovado em 28/11/14 